\title{
The diagnostic and prognostic value of plasma microRNA-125b-5p in patients with multiple myeloma
}

\author{
YANXIA JIANG ${ }^{1}$, YAJING LUAN ${ }^{2}$, HONG CHANG $^{3,4}$ and GUOAN CHEN ${ }^{1}$ \\ ${ }^{1}$ Hematology Department, The 1st Affiliated Hospital of Nanchang University, Nanchang, Jiangxi 330000; \\ ${ }^{2}$ Department of Basic Medical Sciences, Tianjin Medical University, Tianjin 300000, P.R. China; \\ ${ }^{3}$ Division of Molecular and Cellular Biology, Toronto General Research Institute, Toronto, ON M5G 2C4; \\ ${ }^{4}$ Department of Laboratory Medicine and Pathobiology, University of Toronto, Toronto, ON M5S 1A1, Canada
}

Received November 7, 2017; Accepted May 31, 2018

DOI: $10.3892 / 01.2018 .9128$

\begin{abstract}
Aberrant expression of microRNAs (miRNAs) contributes to the progression and outcomes of several types of tumor, while circulating miRNAs have been reported to act as biomarkers for several types of cancer. To identify specific circulating miRNAs associated with multiple myeloma (MM), a miRNA microarray analysis was used, which identified 8 upregulated miRNAs and 4 downregulated miRNAs in the plasma of 6 patients with MM compared with 6 healthy individuals. Based on the microarray results, the 8 miRNAs (miR-125b-5p, miR-483-3p, miR-4326, miR-6894-3p, miR-4498, miR-490-3p, miR-7155-5p and miR-937-3p), which were notably upregulated in MM patients were chosen for a second clinical study in 20 healthy controls and 35 patients with MM using reverse transcription- quantitative polymerase chain reaction. Receiver operating characteristic analysis demonstrated that miR-125b-5p and miR-490-3p displayed considerable diagnostic accuracy for $\mathrm{MM}$ with areas under the curve of $0.954(\mathrm{P}<0.001)$ and $0.866(\mathrm{P}=0.028)$, respectively. In addition, the plasma level of miR-125b-5p was associated with the international staging system disease stage. Patients with higher levels of plasma miR-125b-5p had a significantly shorter event-free survival. However, miR-490-3p levels were not associated with event-free survival $(\mathrm{P}>0.05)$. In summary, miR-125b-5p may serve as a potential clinical biomarker for MM.
\end{abstract}

\section{Introduction}

Multiple myeloma (MM) is a heterogeneous tumor featured by infiltration of the bone marrow by malignant plasma cells. It ranks as the second most prevalent disease among the yet

Correspondence to: Dr Guoan Chen, Hematology Department, The 1st Affiliated Hospital of Nanchang University, 17 Yongwaizheng Street, Donghu, Nanchang, Jiangxi 330000, P.R. China

E-mail: guoanchen010@sina.com

Key words: circulating microRNA, multiple myeloma, biomarker incurable hematologic malignancies, with survival durations ranging from a few months to more than 10 years $(1,2)$. Although the mortality of MM has decreased because of the development of high-dose therapy and innovative agents such as bortezomib, thalidomide and lenalidomide, long-term prognosis remains poor (3). Early diagnosis and treatment can slow disease progression and enhance prognosis, but patients with late-stage disease are usually unresponsive to therapeutic interventions. Therefore, finding noninvasive biomarkers for the detection of cancer may be beneficial for patient survival. Some studies have revealed that molecular markers, including circulating microRNAs (miRNAs), can be useful in diagnosing and monitoring tumors $(4,5)$. Therefore, a highly sensitive and specific noninvasive biomarker that diagnoses MM and assists in determining the prognosis for patients is needed.

miRNAs are noncoding RNA molecules approximately 19-25 nucleotides in length. miRNAs suppress mRNA translation and gene expression by pairing with the 3'-untranslated region (3'UTR) of target mRNAs (6). Since distinct miRNA expression determines the signatures of various cancers, miRNAs may have strong association with cancer pathogenesis and progression (7). Indeed, abnormal miRNA expression, which has been found in MM, leads to carcinogenesis and cancer progression by promoting oncogene expression $(8,9)$. Earlier studies have revealed that miR-125b-5p, which is upregulated in different types of cancers, may belong to a novel class of oncogenes $(10,11)$. However, most of those studies focused on tissue- or cell-based miR-125b-5p expression. To make use of this noninvasive biomarker, the diagnostic and prognostic value of circulating miRNA-125b-5p in patients with MM must be ascertained.

The purpose of this study was to probe into the differential expression (DE) of global miRNAs in the plasma of MM patients and to compare with miRNAs in healthy individuals. We used an miRNA microarray analysis to determine the expression of eight miRNAs (miR-125b-5p, miR-483-3p, miR-4326, miR-6894-3p, miR-4498, miR-490-3p, miR-7155-5p, and miR-937-3p) that are notably increased in MM patients and compared them to the levels in healthy individuals. By quantitatively analyzing these miRNAs in a second clinical study, we discovered that the levels of five 
miRNAs (miR-125b-5p, miR-4326, miR-4498, miR-490-3p, and miR-7155-5p) were increased in the plasma of all patients. Receiver operating characteristic (ROC) analysis illustrated that miR-125b-5p and miR-490-3p displayed considerable diagnostic accuracy for MM. Moreover, plasma miR-125b-5p levels were correlated with shorter event-free survival (EFS). Therefore, this study determined the diagnostic value of circulating miRNAs and identified whether miRNAs could be potentially prognostic biomarkers for MM.

\section{Materials and methods}

Tissue specimens and patient characteristics. The specimens in this study were obtained from 41 patients who were diagnosed in accordance with the National Comprehensive Cancer Network (NCCN) clinical practice guidelines for MM at the 1st Affiliated Hospital of Nanchang University from October 2015 to July 2017 (12). Among the 41 plasma specimens from MM patients, 6 were used for microarray analysis, and 35 served as the validation set. All patients who receiving chemotherapy and/or biotherapy were excluded, and patients with other types of malignant tumors were also eliminated. From healthy controls, we used 20 plasma samples in the validation set, who range in age from 17 to 63.20 healthy individuals were recruited to the 1st Affiliated Hospital of Nanchang University between October 2015 to July 2017, including 8 males and 12 females. Venous blood was collected into tubes with EDTA (BD Biosciences, Franklin Lakes, NJ, USA). Plasma was transferred to a sterile tube and then immediately stored at $-80^{\circ} \mathrm{C}$ after being frozen in liquid nitrogen. The study was approved by the Medical Research Ethics Committee of The First Affiliated Hospital of Nanchang University (Nanchang, China), and written informed consent was obtained from all study subjects. Additionally, FISH analysis was performed in 35 patients. Purified MM patient PCs $\left(\sim 10^{5}\right.$ cells) were classified using the FISH technique. The following probe sets obtained from Vysis (Abbott Diagnostics, Berkshire, UK) were used for classification. D13S319 Spectrum-Orange/LSI-13q34-Spectrum-Green (del(13q) probe), IgH Spectrum-Green/FGFR3 Spectrum-Orange ( $\mathrm{t}(4 ; 14)$ fusion probe), IgH Spectrum-Green/CCND1 Spectrum-Orange $(\mathrm{t}(11 ; 14)$ fusion probe), IgH Spectrum-Green/MAF Spectrum-Orange $(\mathrm{t}(14 ; 16)$ fusion probe), and IgH dualcolor (Sperctrum-Green/Spectrum-Orange) break-apart probe. At least 100 nuclei were scored for each probe. 35 MM patients were cytogenetically classified using FISH, then we investigate the miR-125b-5p expression in the different genetic subtypes of MM patients. The $35 \mathrm{MM}$ patient samples were from the original cohort of 41 patients with MM that were recruited to the study. The patients details are described in Table I.

miRNA array. Total RNA was extracted from samples from $6 \mathrm{MM}$ patients and 6 healthy controls using phenol-chloroform (TRIzol; Invitrogen; Thermo Fisher Scientific, Inc., Waltham, MA, USA). The quality of RNA was assessed by capillary electrophoresis (Agilent Technologies, Inc., Santa Clara, CA, USA). Libraries for small RNA sequencing were prepared using NEB kits (New England Biolabs, Inc., Ipswich, MA, USA). Libraries were quantified using Agilent Bioanalyzer 2100 system by DNA high sensitivity chips. Clean reads 21-22 nucleotides in length were screened for miRNAs and mapped onto a reference genome with Bowtie. The functions of new miRNAs were analyzed by miRDeep2 software. DE sequence was used to quantify differentially expressed miRNAs and to assess statistical significance.

Validation of plasma miRNAs by $q R T-P C R$. TRIzol reagent (Invitrogen; Thermo Fisher Scientific, Inc.) was used to extract total RNA. cDNA was reverse transcribed with the M-MLV Reverse Transcriptase kit (GeneCopoeia, Rockville, MD, USA) following the manufacturer's protocol. Approximately 1,200 $\mu 1$ plasma was employed for miRNA isolation. We employed quantitative reverse transcription PCR (qRT-PCR) with SYBR-Green (Takara, Osaka, Japan) to detect miR-125b-5p, miR-483-3p, miR-4326, miR-6894-3p, miR-4498, miR-490-3p, miR-7155-5p, and miR-937-3p expression levels, U6 was used as an internal control. The reaction was performed via 40 amplification cycles using the following protocol: $95^{\circ} \mathrm{C}$ for $3 \mathrm{~min}, 95^{\circ} \mathrm{C}$ for $45 \mathrm{sec}, 55^{\circ} \mathrm{C}$ for $15 \mathrm{sec}$, and $72^{\circ} \mathrm{C}$ for $50 \mathrm{sec}$. There was no significant difference in the U6 $\mathrm{Ct}$ value between control and $\mathrm{MM}$ samples. The $\Delta \mathrm{Ct}=$ average $\mathrm{Ct}$ (miRNA of target)-average $\mathrm{Ct}$ (U6). The primers used in PCR were as follows: miR-125b-5p forward, 5'-TGCGCT CCCTGAGACCCTAAC-3' and reverse, 5'-CCAGTGCAG GGTCCGAGGTATT-3'; miR-483-3p forward, 5'-TGCGCT CACTCCTCTCCTCCC-3' and reverse, 5'-CTCAAGTGT CGTGGAGTCGGCAA-3'; miR-4326 forward, 5'-GCCCGC TGTTCCTCTGTCTCCC-3' and reverse, 5'-GTGCAGGGT CCGAGGT-3'; miR-6894-3p forward, 5'-TGCGCTTGCCTG CCCTCTTCC-3' and reverse, 5'-CTCAAGTGTCGTGGA GTCGGCAA-3'; miR-4498 forward, 5'-TGCGCTGGGCTG GTAGGGCAAG-3' and reverse, 5'-CTCAAGTGTCGTGGA GTCGGCAA-3'; miR-490-3p forward, 5'-TGCGCCAACCTG GAGGATCCA-3' and reverse, 5'-CTCAAGTGTCGTGGA GTCGGCAA-3'; miR-7155-5p forward, 5'-TGCGCTCTG GGGTCTTGGG-3' and reverse, 5'-CTCAAGTGTCGTGGA GTCGGCAA-3'; miR-937-3p forward, 5'-TGCGCATCCGCG CTCTGACTCT-3' and reverse, 5'-CTCAAGTGTCGTGGA GTCGGCAA-3'; and U6 forward, 5'-CTCGCTTCGGCA GCACA-3' and reverse, 5'-AACGCTTCACGAATTTGCG-3'. Samples were analyzed in triplicate, and gene expression was quantified by normalizing target gene expression to that of the internal control using the $2-^{\Delta \Delta \mathrm{Ct}}$ formula.

Statistical analysis. Data were analyzed using SPSS 19.0 software. All assays were repeated three times, and data were represented as the mean $\pm \mathrm{SD}$. Two-tailed t-tests were performed to compare the difference in plasma miRNA expression levels. Student's t-test was used to determine the significance of difference between two groups, and ROC curves were constructed to evaluate the prediction ability of the miRNAs for MM patients. EFS curves were constructed using the Kaplan-Meier method and compared using the log-rank test. $\mathrm{P}<0.05$ was considered to indicate a statistically significant difference.

\section{Results}

miRNA microarray analysis. We carried out an miRNA microarray analysis to screen candidate plasma miRNAs in 
Table I. Clinical characteristics of patients with MM used for reverse transcription-quantitative polymerase chain reaction analysis of plasma samples $(n=35)$.

\begin{tabular}{lc}
\hline Characteristic & $\mathrm{n}(\%)$ \\
\hline Sex & \\
Male & $23(65.7)$ \\
Female & $12(34.3)$ \\
Age range (years) & $35-75$ \\
Mean age (years) & 59 \\
Durie-salmon stage & \\
I & $10(28.57)$ \\
II & $6(17.14)$ \\
III & $19(54.29)$ \\
Karyotype & \\
t(4:14) & $9(25.71)$ \\
( $11: 14)$ & $5(14.28)$ \\
t(14:16) & $6(17.14)$ \\
Del(13q) as a unique abnormality & $10(28.57)$ \\
Normal FISH & $5(14.29)$ \\
\hline
\end{tabular}

MM, multiple myeloma; miR, microRNA.

Table II. Association between miR-125b-5p expression and the clinical pathological characteristics of patients with MM.

\begin{tabular}{lrrr}
\hline Charactaristic & Cases & $\begin{array}{c}\text { miR-125b-5p } \\
\text { expression }\end{array}$ & P-value \\
\hline Age (years) & & & 0.241 \\
$\quad \leq 50$ & 16 & $1.66 \pm 0.03$ & \\
$>50$ & 19 & $1.72 \pm 0.05$ & \\
Sex & & & 0.439 \\
$\quad$ Male & 23 & $1.68 \pm 0.03$ & \\
Female & 12 & $1.76 \pm 0.03$ & \\
Durie-salmon stage & & & 0.009 \\
$\quad$ I/II & 16 & $1.62 \pm 0.04$ & \\
$\quad$ III & 19 & $1.76 \pm 0.03$ & \\
Extramedullary infltration & & & 0.012 \\
$\quad$ Yes & 8 & $1.82 \pm 0.05$ & \\
$\quad$ No & 27 & $1.66 \pm 0.03$ & \\
Karotype & & & 0.455 \\
$\quad$ Normal FISH & 5 & $1.65 \pm 0.12$ & \\
Abnormal FISH & 30 & $1.72 \pm 0.03$ & \\
\hline
\end{tabular}

MM, multiple myeloma; miR, microRNA.

the treatment group (MM patients) and the control group. After data processing and analysis, we identified a set of 12 miRNAs that were differentially expressed between the treatment group (MM patients) and the control group, among which, 8 were significantly upregulated, and 4 were significantly downregulated (Fig. 1).
Detection of differentially expressed miRNA by $q R T-P C R$. To further validate the differentially expressed miRNA identified by the miRNA microarray analysis, we used qRT-PCR to confirm the eight elevated miRNAs (miR-125b-5p, miR-483-3p, miR-4326, miR-6894-3p, miR-4498, miR-490-3p, miR-7155-5p, and miR-937-3p) in plasma samples from $35 \mathrm{MM}$ patients and 20 healthy individuals. The results suggested that the plasma levels of five miRNAs, namely, miR-125b-5p, miR-4326, miR-4498, miR-490-3p and miR-7155-5p (Fig. 2), were significantly increased between the two groups. In addition, miR-125b-5p expression was associated with extramedullary infiltration and was markedly higher in stage III patients $(1.76 \pm 0.03)$ than in stage I/II patients $(1.62 \pm 0.04)$ (both $\mathrm{P}<0.05)$. However, miR-125b-5p expression was not correlated with patient age, sex or karyotype (all $\mathrm{P}>0.05$ ) (Table II).

miR-125b-5p and miR-490-3p can serve as diagnostic tools in $M M$. To compare the diagnostic value of miRNAs with that of typical tumor markers such as carcinoembryonic antigen (CEA) and cancer antigen 199 (CA199), we constructed ROC curves to validated plasma miRNAs in identifying MM patients. As shown in Fig. 3, miR-125b-5p and miR-490-3p were accurate in distinguishing MM patients from healthy controls. The area under the curve (AUC) for miR-125b-5p was 0.954 , with $86 \%$ sensitivity and $96 \%$ specificity [95\% confidence interval (CI) 0.901-1.006; P<0.001]; the AUC for miR-490-3p was 0.866 , with $60 \%$ sensitivity and $85 \%$ specificity $(95 \% \mathrm{CI}$ 0.522-0.836; $\mathrm{P}=0.028)$.

Prognostic value of plasma miRNA expression in MM. We further assessed the correlation between miRNA expression and MM prognosis by Kaplan-Meier analysis. MM patients receive the chemotherapy of bortezomib, thalidomide plus dexamethasone (VTD) regimen, followed by thalidomide maintenance therapy. MM patients were divided into either high or low miR-125b-5p/miR-490-3p expression subgroups. Using this approach, we compared EFS between high- and low-expression subgroups. EFS durations were calculated from the time of diagnosis to the date of relapse or death. After a median follow-up 9.5 months (range, 1-26 months) from diagnosis, the median EFS duration in patients expressing high levels of miR-125b-5p was 8 months, and that in patients expressing low levels of miR-125b-5p was 13 months. Our results indicated that patients with higher miR-125b-5p expression had a shorter EFS than patients with lower miR-125b-5p expression ( $\mathrm{P}=0.02$, Fig. 4). However, there was no difference between high and low miR-490-3p expression groups, with median EFS of 12 and 13 months, respectively ( $\mathrm{P}=0.23$; Fig. 4$)$.

\section{Discussion}

Plasma miRNAs have been previously reported in the context of some cancers $(4,13,14)$. In the present study, we performed an miRNA microarray analysis to elucidate the expression profiles of plasma miRNA in six MM patients and six healthy individuals. In accordance with the microarray analysis, we found that the levels of eight differentially expressed miRNAs were significantly higher in MM patients than in healthy 


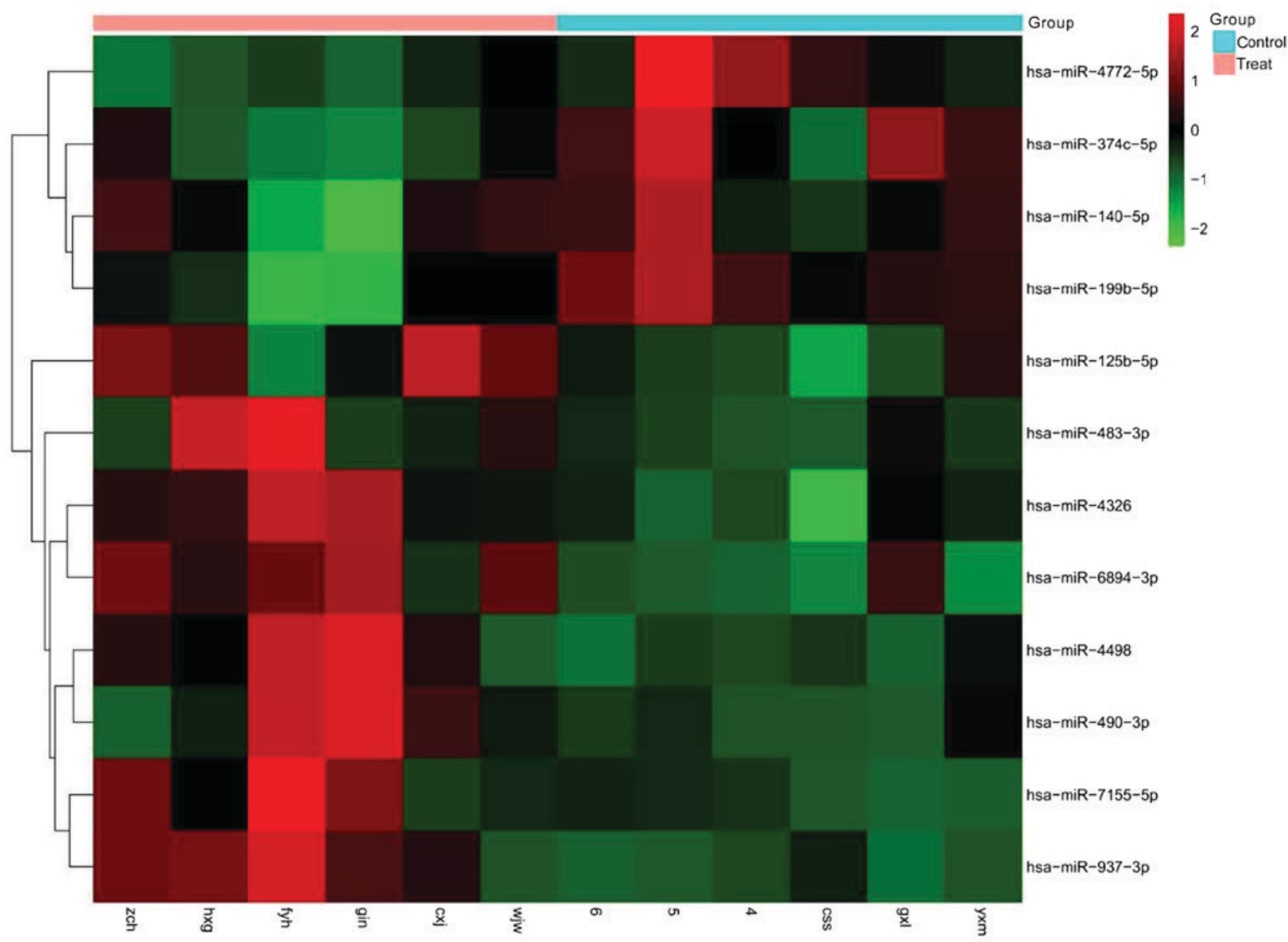

Figure 1. Dysregulation of plasma miRNAs in multiple myeloma as determined by microarray analysis. miR, microRNA.
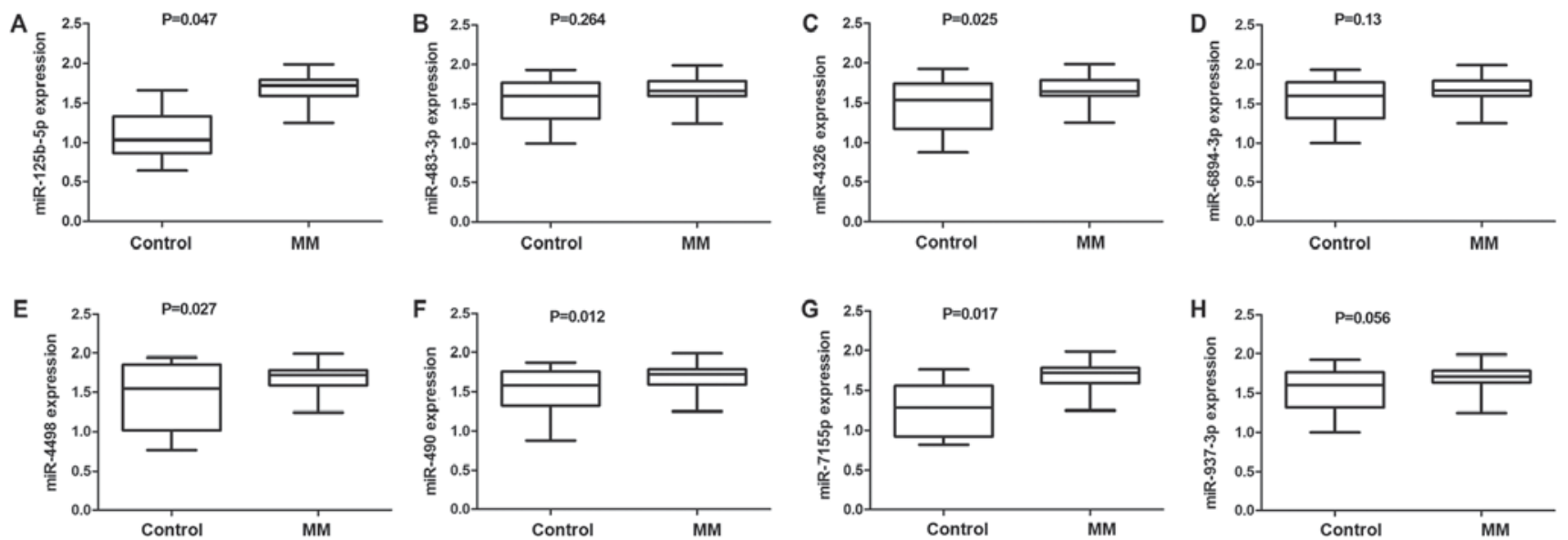

Figure 2. miRNA expression in clinical samples as determined by reverse transcription- quantitative polymerase chain reaction. The expression levels of (A) miR-125b-5p, (B) miR-483-3p, (C) miR-4326, (D) miR-6894-3p, (E) miR-4498, (F) miR-490-3p, (G) miR-7155-5p and (H) miR-937-3p in MM (n=35) and control $(\mathrm{n}=20)$ samples. MM, multiple myeloma; miR, microRNA.

individuals. We then validated the elevated levels of five of those (miR-125b-5p, miR-4326, miR-4498, miR-490-3p and miR-7155-5p) in a second clinical study. Most of the targets of these miRNAs are cancer-associated genes such as APC2, IGF2, and IRF4 (15-17) that are involved in cell proliferation, cell cycle, apoptosis, invasion and metastasis.

Furthermore, ROC analysis demonstrated that miR-125b-5p and miR-490-3p were accurate in distinguishing
MM patients from healthy subjects, resulting in AUCs of 0.954 (sensitivity $=86 \%$, specificity $=96 \%, 95 \%$ CI $0.901-1.006$, $\mathrm{P}<0.001$ ) and 0.866 (sensitivity $=60 \%$, specificity $=85 \%, 95 \% \mathrm{CI}$ $0.522-0.836, \mathrm{P}=0.028$ ), respectively. However, no significant association was found between miR-490-3p and EFS of MM patients. Moreover, our data showed that patients with higher miR-125b-5p expression had a shorter EFS. Additionally, miR-125b-5p levels were correlated with international staging 

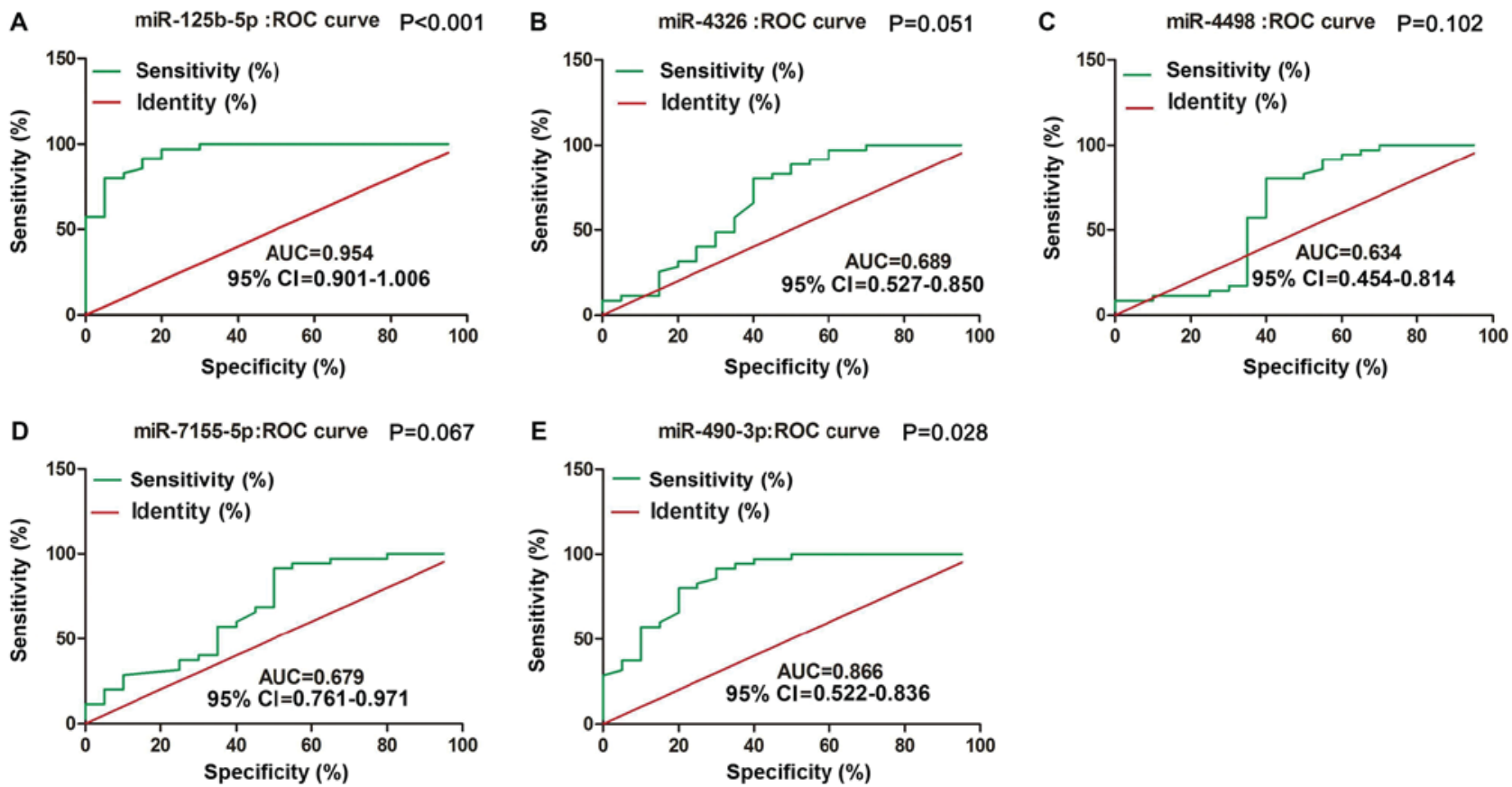

Figure 3. ROC curve analysis using validated plasma miRNAs for identifying patients with MM. miR-125b-5p and miR-490-3p display considerable diagnostic accuracy based on ROC analysis. (A) The AUC for miR-125b-5p was $0.954(\mathrm{P}<0.001)$ indicating considerable diagnostic accuracy. (B) The AUC for miR-4362 was $0.689(\mathrm{P}=0.051)$. (C) The AUC for miR-4489 was 0.634 ( $\mathrm{P}=0.102)$. (D) The AUC for miR-7155-5p was 0.679 ( $\mathrm{P}=0.067)$. (E) The AUC for miR-490-3p was $0.866(\mathrm{P}=0.028)$, indicating considerable diagnostic accuracy. AUC, area under the curve; miR, microRNA; CI, confidence interval; ROC, receiver operating characteristic.
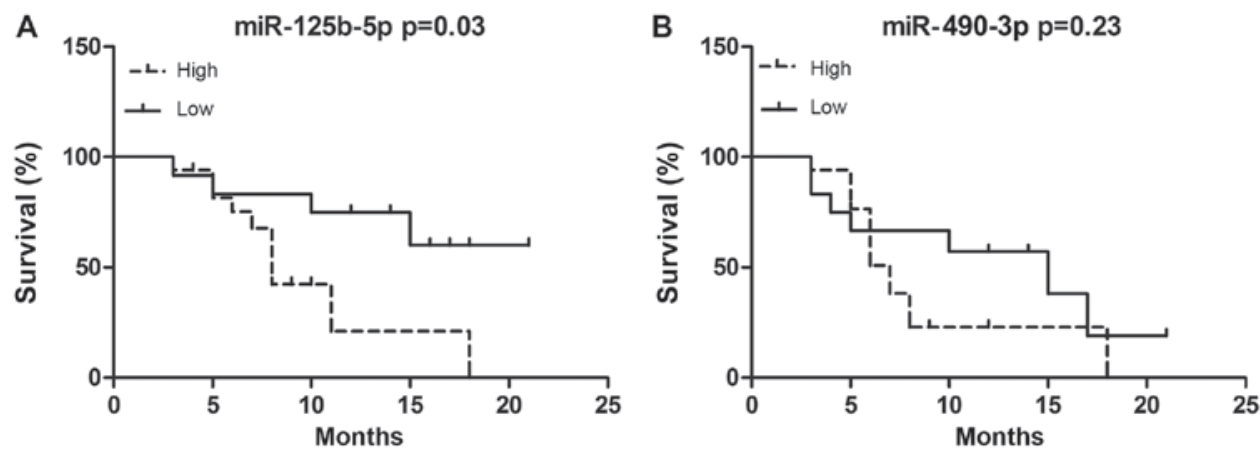

Figure 4. Association between miRNA levels and event-free survival in patients with multiple myeloma as determined by Kaplan-Meier survival analysis. (A) miR-125b-5p and (B) miR-490-3p. miR, microRNA.

system (ISS) stage, thereby indicating the diagnostic value of miR-125b-5p.

Deregulation of miR-490-3p has been observed in many types of cancers, such as lung cancer (18), colorectal cancer (19) and gastric cancer (20). Zhang et al demonstrated that the urinary level of miR-490 is positively associated with focal segmental glomerulosclerosis (FSGS) disease activity, and thus miR-490 might be a promising biomarker for evaluating FSGS disease activity (21). In this study, miR-490 was overexpressed in MM patient samples and could thus be used as an early tumor biomarker. miR-125b-5p has been demonstrated to act as either cancer promoter or cancer suppressor in different types of cancer. Upregulation of miR-125b-5p has been detected in pancreatic cancer (22), prostate cancer (23), and acute myeloid leukemia (24), while downregulation of miR-125b-5p has been observed in thyroid cancer (25) and oral squamous cell carcinoma (26). miR-125b-5p has also been found to promote tumor cell proliferation and inhibit p53-dependent apoptosis in human neuroblastoma cells (27). Several studies indicate that p53 is the direct target of miR-125b, and MM cells responding to dexamethasone exhibit enhanced expression of the oncogenic miR-125b $(28,29)$. However, the mechanism by which miR-125b regulates MM remains unknown. Importantly, circulating miR-125b-5p has been reported to be correlated with colorectal cancer (30), breast cancer (31) and hepatocellular carcinoma (32). Circulating and serum-derived miRNAs have also been identified in MM patients (33). In our study, plasma miR-125b-5p levels were correlated with extramedullary infiltration and ISS stage in MM patients. Therefore, we concluded that plasma miR-125b-5p may be correlated with poor prognosis in MM patients.

Circulating serum/plasma miRNA expression profiles have been identified and used as noninvasive biomarkers for tumor identification and prognosis (34). Furthermore, some 
circulating miRNAs may be correlated with chemotherapeutic resistance in tumors $(31,35)$. Therefore, the expression profiles of circulating miRNAs can be used to monitor disease progression. To the best of our knowledge, this is the first study to explore the relevance of plasma miR-125b-5p to MM. We demonstrated that miR-125b-5p can be used as a feasible biomarker with significant accuracy in diagnosing patients with MM. Nevertheless, there are several limitations in our study. First, our sample size is small, and long-term follow-up is required to validate the relationship between miR-125b-5p levels and patient outcomes. In addition, large-scale and in-depth studies are necessary. Second, the potential mechanisms of miR-125b-5p in MM were not elucidated.

In conclusion, this study demonstrated that circulating miR-125b-5p levels can be used as a diagnostic and predictive biomarker in MM. miR-125b-5p can be used to identify MM patients with poor prognosis and aid in the selection of timely comprehensive therapy.

\section{Acknowledgements}

Not applicable.

\section{Funding}

International Collaboration Fund from National Science and Technology Committee of China (grant no. 2011DFA32820). The National Natural Science Fund Project (grant no. 81460037 and 81760040). The National Science Foundation for Young Scientists of China (grant no. 81600180). Innovation Fund Project in Jiangxi Province (grant no. YC2016-B018).

\section{Availability of data and materials}

The datasets used during the current study are available from the corresponding author on reasonable request.

\section{Authors' contributions}

YJ and YL performed the molecular biology experiments and drafted the manuscript. HC performed the statistical analysis. GC conceived the study and participated in its design and helped to draft the manuscript.

\section{Ethics approval and consent to participate}

The present study was approved by the Medical Research Ethics Committee of The First Affiliated Hospital of Nanchang University (Nanchang, China) and written informed consent was obtained from all study participants.

\section{Consent for publication}

Not applicable.

\section{Competing interests}

The authors declare that they have no competing interests.

\section{References}

1. Sonneveld P, Avet-Loiseau H, Lonial S, Usmani S, Siegel D, Anderson KC, Chng WJ, Moreau P, Attal M, Kyle RA, et al: Treatment of multiple myeloma with high-risk cytogenetics: A consensus of the International myeloma working group. Blood 127: 2955-2962, 2016.

2. Abdi J, Chen $\mathrm{G}$ and Chang H: Erratum: Drug resistance in multiple myeloma: Latest findings and new concepts on molecular mechanisms. Oncotarget 6: 7364, 2015.

3. Mimura N, Hideshima T and Anderson KC: Novel therapeutic strategies for multiple myeloma. Exp Hematol 43: 732-741, 2015.

4. Liao TL, Chen YM, Hsieh CW, Chen HH, Lee HC, Hung WT, Tang KT and Chen DY: Upregulation of circulating microRNA-134 in adult-onset Still's disease and its use as potential biomarker. Sci Rep 7: 4214, 2017.

5. Pilli T, Cantara S, Marzocchi C, Cardinale S, Santini C, Cevenini $G$ and Pacini F: Diagnostic value of circulating microRNA-95 and -190 in the differential diagnosis of thyroid nodules: A validation study in 1000 consecutive patients. Thyroid 27: 1053-1057, 2017.

6. Bartel DP: MicroRNAs: Genomics, biogenesis, mechanism, and function. Cell 116: 281-297, 2004.

7. Zhang B, Pan X, Cobb GP and Anderson TA: microRNAs as oncogenes and tumor suppressors. Dev Biol 302: 1-12, 2007.

8. Zheng P, Guo H, Li G, Han S, Luo F and Liu Y: PSMB4 promotes multiple myeloma cell growth by activating NF- $\kappa \mathrm{B}-\mathrm{miR}-21$ signaling. Biochem Biophys Res Commun 458: 328-333, 2015.

9. Shen X, Guo Y, Yu J, Qi J, Shi W, Wu X, Ni H and Ju S: miRNA-202 in bone marrow stromal cells affects the growth and adhesion of multiple myeloma cells by regulating B cell-activating factor. Clin Exp Med 16: 307-316, 2016.

10. Shi XB, Xue L, Yang J, Ma AH,Zhao J, Xu M,TepperCG,Evans CP, Kung HJ and deVere White RW: An androgen-regulated miRNA suppresses Bak1 expression and induces androgen-independent growth of prostate cancer cells. Proc Natl Acad Sci USA 104: 19983-19988, 2007.

11. Bousquet M, Quelen C, Rosati R, Mansat-De Mas V, La Starza R, Bastard C, Lippert E, Talmant P, Lafage-Pochitaloff M, Leroux D, et al: Myeloid cell differentiation arrest by miR-125b-1 in myelodysplastic syndrome and acute myeloid leukemia with the $\mathrm{t}(2 ; 11)$ (p21;q23) translocation. J Exp Med 205: 2499-2506, 2008.

12. Anderson KC: Progress and paradigms in multiple myeloma. Clin Cancer Res 22: 5419-5427, 2016.

13. Riazalhosseini B, Mohamed R, Apalasamy YD, Langmia IM and Mohamed Z: Circulating microRNA as a marker for predicting liver disease progression in patients with chronic hepatitis B. Rev Soc Bras Med Trop 50: 161-166, 2017.

14. Wu L, Hu B, Zhao B, Liu Y, Yang Y, Zhang L and Chen J: Circulating microRNA-422a is associated with lymphatic metastasis in lung cancer. Oncotarget 8: 42173-42188, 2017.

15. Xu G, Zhang Z, Zhang L, Chen Y, Li N, Lv Y, Li Y and Xu X: miR-4326 promotes lung cancer cell proliferation through targeting tumor suppressor APC2. Mol Cell Biochem 443: $151-157,2018$.

16. Bertero T, Gastaldi C, Bourget-Ponzio I, Imbert V, Loubat A, Selva E, Busca R, Mari B, Hofman P, Barbry P, et al: miR-483-3p controls proliferation in wounded epithelial cells. FASEB J 25: 3092-3105, 2011.

17. Morelli E, Leone E, Cantafio ME, Di Martino MT, Amodio N, Biamonte L, Gullà A, Foresta U, Pitari MR, Botta C, et al: Selective targeting of IRF4 by synthetic microRNA-125b-5p mimics induces anti-multiple myeloma activity in vitro and in vivo. Leukemia 29: 2173-2183, 2015.

18. Gu H, Yang T, Fu S, Chen X, Guo L and Ni Y: MicroRNA-490-3p inhibits proliferation of A549 lung cancer cells by targeting CCND1. Biochem Biophys Res Commun 444: 104-108, 2014.

19. Zheng K, Zhou X, Yu J, Li Q, Wang H, Li M, Shao Z, Zhang F, Luo Y, Shen Z, et al: Epigenetic silencing of miR-490-3p promotes development of an aggressive colorectal cancer phenotype through activation of the Wnt/ $\beta$-catenin signaling pathway. Cancer Lett 376: 178-187, 2016.

20. Zhou B, Wang Y, Jiang J, Jiang H, Song J, Han T, Shi J and Qiao H: The long noncoding RNA colon cancer-associated transcript-1/miR-490 ax is regulates gastric cancer cell migration by targeting hnRNPA1. IUBMB Life 68: 201-210, 2016. 
21. Zhang W, Zhang C, Chen H, Li L, Tu Y, Liu C, Shi S, Zen K and Liu Z: Evaluation of microRNAs miR-196a, miR-30a-5P, and miR-490 as biomarkers of disease activity among patients with FSGS. Clin J Am Soc Nephrol 9: 1545-1552, 2014.

22. Bloomston M, Frankel WL, Petrocca F, Volinia S, Alder H, Hagan JP, Liu CG, Bhatt D, Taccioli C and Croce CM: MicroRNA expression patterns to differentiate pancreatic adenocarcinoma from normal pancreas and chronic pancreatitis. JAMA 297 1901-1908, 2007.

23. Ma Q, Chen Z, Jia G, Lu X, Xie X and Jin W: The histone demethylase PHF8 promotes prostate cancer cell growth by activating the oncomiR miR-125b. Onco Targets Ther 8 : 1979-1988, 2015

24. Liu J, Guo B, Chen Z, Wang N, Iacovino M, Cheng J, Roden C, Pan W, Khan S, Chen S, et al: miR-125b promotes MLL-AF9-driven murine acute myeloid leukemia involving a VEGFA-mediated non-cell-intrinsic mechanism. Blood 129 1491-1502, 2017.

25. Visone R, Pallante P, Vecchione A, Cirombella R, Ferracin M, Ferraro A, Volinia S, Coluzzi S, Leone V, Borbone E, et al: Specific microRNAs are downregulated in human thyroid anaplastic carcinomas. Oncogene 26: 7590-7595, 2007.

26. Veerla S, Lindgren D, Kvist A, Frigyesi A, Staaf J, Persson H, Liedberg F, Chebil G, Gudjonsson S, Borg A, et al: MiRNA expression in urothelial carcinomas: important roles of miR-10a, miR-222, miR-125b, miR-7 and miR-452 for tumor stage and metastasis, and frequent homozygous losses of miR-31. Int J Cancer 124: 2236-2242, 2009

27. Xia HF, He TZ, Liu CM, Cui Y, Song PP, Jin XH and Ma X: MiR-125b expression affects the proliferation and apoptosis of human glioma cells by targeting Bmf. Cell Physiol Biochem 23: $347-358,2009$
28. Le MT, Teh C, Shyh-Chang N, Xie H, Zhou B, Korzh V, Lodish HF and Lim B: MicroRNA-125b is a novel negative regulator of p53. Genes Dev 23: 862-876, 2009.

29. Murray MY, Rushworth SA, Zaitseva L, Bowles KM and Macewan DJ: Attenuation of dexamethasone-induced cell death in multiple myeloma is mediated by miR-125b expression. Cell Cycle 12: 2144-2153, 2013.

30. Chen $\mathrm{H}$ and $\mathrm{Xu} \mathrm{Z}$ : Hypermethylation-associated silencing of miR-125a and miR-125b: A potential marker in colorectal cancer. Dis Markers 2015: 345080, 2015.

31. Wang H, Tan G, Dong L, Cheng L, Li K, Wang Z and Luo H: Circulating MiR-125b as a marker predicting chemoresistance in breast cancer. PLoS One 7: e34210, 2012.

32. Liu W, Hu J, Zhou K, Chen F, Wang Z, Liao B, Dai Z, Cao Y, Fan J and Zhou J: Serum exosomal miR-125b is a novel prognostic marker for hepatocellular carcinoma. Onco Targets Ther 10: 3843-3851, 2017.

33. Wong KY, Li Z, Zhang X, Leung GK, Chan GC and Chim CS: Epigenetic silencing of a long non-coding RNA KIAA0495 in multiple myeloma. Mol Cancer 14: 175, 2015.

34. Jairajpuri DS, Malalla ZH, Mahmood N and Almawi WY: Circulating microRNA expression as predictor of preeclampsia and its severity. Gene 627: 543-548, 2017.

35. Niu J, Xue A, Chi Y, Xue J, Wang W, Zhao Z, Fan M, Yang CH, Shao ZM, Pfeffer LM, et al: Induction of miRNA-181a by genotoxic treatments promotes chemotherapeutic resistance and metastasis in breast cancer. Oncogene 35: 1302-1313, 2016. 